\title{
ARTICLE
}

\section{Controlling the confusion: using barrier analysis in the care home sector $^{\dagger}$}

\author{
Robert Colgate, Karyn Davies, Helen Lambert \& Alison Turner
}

\section{Robert Colgate has been a} consultant in old age psychiatry based at the Princess of Wales Hospital in Bridgend, South Wales, since 1997. His interests include postgraduate (adult) education and risk assessment and management with an emphasis on the mental health of older adults. Karyn

Davies qualified as a registered mental health nurse in 1989. She has specialised in older people's mental health services for about 20 years. During this time she has gained extensive hospital and community experience. Her special interest is education, in particular specialist dementia care education and training. Helen Lambert qualified as a state registered occupational therapist in 1991 Initially worked in the learning disability and acute mental health field, Helen has, for the past 9 years, specialised in older people's mental health services, in particular education and the provision of specialist education and training. Alison Turner qualified as a state registered occupational therapist in 1983. She gained extensive experience working within the learning disability field and has specialised in older people's mental health services for the past 7 years. Alison's special interests are the provision of education in dementia care along with a specialist role within the drugs for dementia team. Correspondence DrRobert Colgate, Princess of Wales Hospital Coity Road, Bridgend CF31 1RO, UK. Email: Robert.Colgate@wales.nhs.uk

tFor a commentary on this article see pp. 434-435, this issue.

\begin{abstract}
SUMMARY
The provision of mental health teaching and training to staff in the care home sector by a dedicated educational team was established in Bridgend in 2003. A multiprofessional old age liaison psychiatry team was created in 2009 specifically to link closely with the teaching staff, further building on previous work on referral coordination and a single point of access. This article describes the application of barrier analysis to deliver both this new service and mental health education to the care home sector. Imaginative uses of information technology and multimedia to support the efforts of all staff are described, as are some pitfalls and hazards to avoid. The most effective elements of these developments are discussed, using barrier analysis as the explanatory model, with an emphasis on aspects that could be adopted more widely.
\end{abstract}

\section{DECLARATION OF INTEREST}

None.

There is now very little room for doubt that the care of older people will be a major consumer of health and social services resources in the UK for the foreseeable future (Banks 2011). Similar pressures, including effective coordination of care delivery with training, have also been identified in other countries (Shaji 2006; Bernstein 2010). A perfectly valid desire to have an individualised care package at home contrasts strongly with the need to achieve economies of scale by providing care for more and more older adults with mental health problems in residential and nursing care settings.

There is a clear need for mental health services for older adults to be closely involved in the monitoring of mental health needs in the care home sector. Rates of depressive illness remain consistently high, at around $40 \%$ in the UK (Godfrey 2005), while the number of cases of dementia is rising steadily as aging populations grow. Published reports (National Institute for Health and Clinical Excellence 2006; All-Party
Parliamentary Group on Dementia 2009) point to widespread lack of mental health expertise in primary care and rising societal expectations to manage increasingly complex combinations of mental and physical health needs.

Over time, these issues have received attention in a wide variety of sources, from conventional healthcare publications to general interest articles in magazines and mass media. Some idea of the range of public interest can be shown by just a few examples.

A collaboration with the $\mathrm{BBC}$ and The Open University by business expert Sir Gerry Robinson led to the production of a film entitled 'Can Gerry Robinson fix Dementia Care Homes?', which is available as a DVD and is promoted for use as a teaching or public education resource (Open University 2010). An anonymous personal view column in the BMJ (Anonymous 2010) catalogues a damaging sequence of admissions and attempts at treatment involving the person's father before concluding that the only reliable source of practical help was 'family, friends and neighbours' - the conclusion is all the more striking as the author is a hospital consultant.

In the Royal College of Psychiatrists' College Seminars series, Jolley et al (1998) encouraged mental health services to 'address the situation (of mental illness in care home settings) actively' by using a genuine multidisciplinary approach to provide a 'full competent assessment'. The Consumers Association magazine Which? recently published an article entitled 'Care homes investigation' describing in some detail the experiences of three actors staying in four care homes in 2011 (Which? 2011). The article contains a six-item checklist of good practice points and sources for further information.

\section{Bridgend mental health service for older adults}

In late 2009, the mental health service for older adults in Bridgend was reorganised to create a dedicated multiprofessional team with specific 
responsibility for residents with mental illness in care homes in the Bridgend County Borough area. This team also maintained responsibility for providing a mental health liaison service to secondary care health services (based at the Princess of Wales and Maesteg General hospitals), while the team consultant also provided senior psychiatric advice to a shared care ward (rehabilitation medicine and mental healthcare) on the Princess of Wales Hospital site.

The All-Party Parliamentary Group on Dementia (2009) has drawn attention to the inadequate expert input, stating that 'the lack of support from specialist mental health services that could provide support and training to staff is [...] another barrier to workforce development' (p. xii). Published evaluations of care home in-reach teams have recognised the potential value of a more focused approach by nursing staff (Lawrence 2010) or nurses working with physiotherapists (Szczepura 2008). Commissioners and planners of local services in Bridgend confirmed that suitable training and education of staff was indeed a key priority area. Jolley et al (1998) concur with this, stating that 'the need for generous and sensitive attention to the training and support of staff cannot be overestimated'. Consequently, a strategic decision was taken to deliberately integrate the new liaison service with a well-established educational team also based in Bridgend.

The educational team collaborates closely with the liaison team to identify further potential training or advisory issues within a care home. This may involve referral of a resident for advice regarding a specific issue or an individual approach to care or intervention. It may also include supportive advice concerning design principles within the care setting or recommended approaches to meaningful interaction - building on material taught previously in educational sessions. Other issues that have been considered include concern about mental capacity, pain assessment and the management, care and support of particular behaviours.

In addition, the educational team has consistently emphasised the need to actively consider the role and purpose of antipsychotic medication in dementia, and it has been able to coordinate its efforts with the members of the care home in-reach team. Most recently, attention has focused on the place of community 'do not attempt resuscitation' (DNAR) orders and the role of mental health staff in providing advice and support to primary care. In selected cases, this has been undertaken with the intention of avoiding an unnecessary or disruptive hospital admission for people in the terminal stages of dementia.

\section{The dementia care training team in Bridgend}

The number of people with dementia in the UK is now about 820000 , which represents about $1.3 \%$ of the overall population (Alzheimer's Research Trust 2010). This figure is predicted to double within 30 years (Department of Health 2009).

Clinical Guideline 42 from the National Institute for Health and Clinical Excellence (2006) makes specific reference to staff training, clearly recommending that:

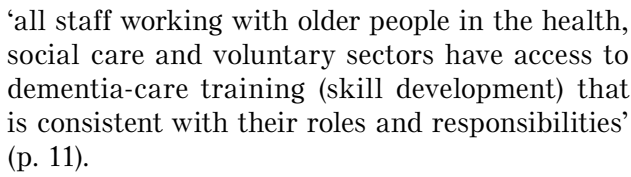
(p. 11).

Prior to this, the need for specialist dementia care training and development had been identified locally in Bridgend through routine practice as well as operational and strategic meetings. As a result, a dementia care training team was established in November 2002 and initially named the Residential Home Advisor Team. The team consists of a full-time registered mental health nurse and two occupational therapists job-sharing the equivalent of one wholetime post.

\section{Aims of the team}

Originally, the aim of the team was to identify and then address the training needs of unqualified care staff working with people with dementia in the Bridgend County Borough area, both in the local authority facilities and throughout the independent care home sector. The team also sought to provide the care home staff with suitable specialist training and education along with playing an advisory role. The mandate of the team has since widened to provide training for other staff groups, including local authority and independent sector domiciliary care and day-centre staff, qualified social workers and community care workers, community- and ward-based mental health staff and medical, surgical and orthopaedic ward staff (both registered and non-registered).

Training sessions have also been held for the carer education programme of Alzheimer's Society (covering the topics of 'What is dementia?', behavioural issues and management of eating and drinking in dementia). Dedicated sessions have been organised for specialist staff in the accident and emergency department and a renal unit as well as for palliative care staff. Most recently, relevant training has been provided for those working in the field of intellectual disability. 


\section{Training needs}

The vision of the team is that wherever a person with dementia is being supported and cared for, within Bridgend County Borough, then those working with them should be appropriately trained and suitably skilled with the expectation that this will lead to enhanced levels of care. This is consistent with the work of Ballard et al (2001) who suggested that staff training is universally seen as a key feature in implementing and maintaining a good standard of care in both nursing and residential care homes. Recent reports continue to highlight the vast need for training of the staff caring for people with dementia.

The All-Party Parliamentary Group on Dementia report Prepared to Care (2009) stated that 'we need to move towards a situation where the workforce as a whole demonstrates effective knowledge and skills in caring for people with dementia' (p. xiv). The report also recommended that, to ensure high-quality dementia care, the process of working between social care and healthcare must be closely integrated: 'standardised training packages would provide quality assurance and consistency' (p. 33). Effective support from specialist mental health teams should also be coordinated with the local education strategy to support the training needs within the care home sector.

\section{Training package}

The dementia care training is a modular training package which has been refined and developed over 9 years and currently consists of nine separate modules (Box 1) delivered over a total of $30 \mathrm{~h}$. The introductory session gives an overview of dementia and the programme then covers a range of issues including communication, legal and ethical matters, behavioural concerns, and hydration and nutrition. In practice, modules are delivered either in a care home to a discrete group of staff from

BOX 1 List of dementia care training modules

- An overview of dementia

- Communication and dementia

- Legal and ethical issues in dementia care

- Physical aspects of dementia care

- Understanding behaviours in dementia

- Positive environments in dementia care

- Meaningful interactions and dementia

- Mental health issues

- Eating and drinking in dementia care

- Pain in dementia that home, or in a training venue (such as a church hall or a community centre) to delegates invited from a number of care homes. Wherever possible, a setting is chosen that is most appropriate to the identified need.

Professional advice and expert opinion has been sought when developing and redesigning the modules to ensure they are evidence based, relevant and contemporary. Each module incorporates a wide variety of teaching and learning strategies that enable and facilitate care staff interaction. The module content and form is also influenced by evaluation responses.

Thetraining packagehas recentlybeen accredited at certificate level with Swansea University. The pilot accredited version commenced in January 2011, with a target group of mental health support workers from across the health board.

Specific 'bespoke' sessions have been written for certain staff groups - for example, an emphasis on strategies to manage pain in dementia was required for medical, surgical and orthopaedic nursing staff. The standard module content and form is also influenced where appropriate by evaluation responses.

The dementia care training team has completed a service evaluation project using an action research model. First-person and cooperative enquiry, plus focus groups and interviews, were the methods chosen to investigate the question 'How does the dementia care training package impact on personcentred practice?' (K. Davies, 2011, personal communication). The data collected showed that the training is generally positively valued and does influence person-centred care. Admirable teacher qualities were identified and the programme was found to be educative and thought-provoking.

\section{Barrier analysis}

The title of this article reflects the complexity involved in providing an effective mental health service to the care home sector and at the same time ensuring an integrated approach to training and education. As part of the design process for the service, several different interventions and innovations were deployed. The measures taken were chosen from within the categories of physical and natural barriers, as described in barrier analysis.

Barrier analysis as a technique was developed in the nuclear and chemical industry during the 1990s primarily to reduce error (Lyons 2004). This approach was subsequently transferred to healthcare settings, again with an emphasis on reducing mistakes. Formal teaching and training in barrier analysis was disseminated widely within 
the National Health Service (NHS) in the UK by the National Patient Safety Agency (NPSA) as a component of root cause analysis.

Using the analysis, barriers can be usefully categorised into four main groups:

1 barriers dependent on people

2 administrative or 'paper' barriers

3 physical or geographical barriers

4 natural barriers related to space or time.

Standard teaching suggests that barriers reliant on paper or on people are weaker and less effective than physical or natural barriers.

To illustrate this, it may be helpful to consider the process of maintaining and improving security at international airports. From first principles, it is a relatively simple task to list several measures and interventions that have progressively developed over recent years to prevent the unauthorised carriage of weapons or hazardous material on commercial flights (Box 2). The list in Box 2 is not meant to be exhaustive, but it does usefully illustrate the essence of barrier analysis. If the items in this list are categorised into one of the four groups - people, administrative, physical or natural - it quickly becomes clear that in this case more attention and investment has been focused on the examples of physical or natural barriers that are traditionally felt to be more effective.

\section{Barrier analysis in healthcare}

Although maybe not as obvious or as dramatic, it may also be helpful to compare the process of maintaining security on commercial flights with common and familiar healthcare processes such as correct site surgery and blood transfusion. In 2008, the World Health Organization introduced a surgical safety checklist (World Alliance for Patient Safety 2008). The first edition incorporated examples of both physical and natural barriers drawn directly from the basic principles of barrier analysis. Strict protocols regarding marking of the site of surgery represent a physical barrier to reduce the incidence of incorrect site surgery. The

BOX 2 Barriers in airport security

- Administrative: printed list of prohibited items, warning signs and notices

- Personnel: routine enquiries from check-in staff

- Physical: checkpoints, including passport control, X-ray screening of hand luggage

- Natural: secure airfield perimeter, separation of open access and 'airside' introduction of a 'time out' period before surgery commences is an excellent example of a natural barrier. Once again, emphasis on physical and natural barriers is entirely consistent with the principles of ensuring a safe and effective process.

Similarly, the adoption of increasingly strict requirements by NHS Wales before a blood transfusion can go ahead represents another attempt to introduce a physical safety barrier. Recent developments include 'zero tolerance' concerning documentation at all stages of blood sampling, matching and administration of blood products or transfusion. Incorrect spelling or missing information will result in rejection of samples for cross-matching.

Although barrier analysis has traditionally been used to explore and analyse adverse incidents after they have happened, the NPSA has identified a prospective role for this technique, searching for possible weaknesses or areas of vulnerability. For the purposes of this article, it is also constructive to use the barrier analysis classifications to explore the issue of mental health supervision of residents in the care home sector.

\section{Development of the liaison service to the care home sector}

In a previous article in this journal, we described the establishment and development of a process of referral coordination based on a single point of access and a dedicated referral coordinator (Colgate 2007). One of the core principles of this process is that patients are prioritised according to need (rather than the availability of staff). Another core principle is that specialist services retain the responsibility for specialist decisions, in this case mental health decisions about mental health need.

New referrals to the mental health service and requests for psychiatric reviews within the care home sector are still closely linked to this established referral coordination process. There is little doubt that the level of mental health need of this group will increase incrementally over time associated with a rising older adult population. Effective communication is maintained using standard templates and distribution of referrals via email.

\section{Background}

In the Bridgend County Borough area, there are currently 25 residential and nursing care homes ranging from small residential care facilities to a large specialist mental health nursing home. Overall, the county has provision of more than 700 care beds. Local data have established that about a third of the residents in this care sector 
are prescribed antipsychotic medication for the treatment of mental illness and a further third have additional mental health needs ranging from relatively mild depressive symptoms to highly complex physical and mental health needs.

At any one time, between 15 and 20 patients in this group have been subject to provisions of the Mental Health Act 1983, and a similar number of (usually standard) authorisations have been in place under the Deprivation of Liberty Safeguards. This substantial workload requires a systematic and robust review process which is still sufficiently flexible to adapt to rapid changes in care needs. The key to achieving this in a reliable and effective manner has been the creation of a spreadsheet for each care home listing the residents with mental health needs in each facility, with sufficient basic information to allocate how often the resident should be reviewed. (This logistic exercise can be viewed as a physical intervention in terms of barrier analysis.)

\section{The review process}

During the initial weeks following establishment of the team, arrangements were made with each care home for several team members to visit together to identify (and review) all the residents in the home who were already known to the mental health service. These data formed the core of the spreadsheet. In most but not all cases, the residents were either on existing community mental health nursing case-loads or regularly attending local mental health out-patient clinics.

In addition, a list of 'drugs of interest' was compiled using the British National Formulary essentially Sections 4.2.1 and 4.2.2 (antipsychotic drugs and antipsychotic depot injections respectively), Section 4.2.3 (antimanic drugs specifically preparations of lithium) and Section 4.11 (drugs for dementia) (British Medical Association 2010). In a modern mental health service, prescription of antipsychotic medication, lithium carbonate (or citrate) as maintenance treatment or any of the drugs for dementia will be associated with at least some degree of mental health need.

The list of 'drugs of interest' was given to care home managers and senior care staff, with the advice that any resident receiving a regular prescription of any of these drugs of interest should either be under regular mental health follow-up or be offered a new mental health assessment. This aspect was also supported by a clinical audit cycle focusing on antipsychotic prescribing.

In our experience, a small number of residents had reliable existing arrangements with local learning disability or general adult psychiatry services. The new liaison team did not interfere with these arrangements. However, a small but significant number of residents with clear psychiatric needs were not under any form of regular mental health follow-up. In most cases, sensitive discussion and negotiation with the relevant general practitioner and care home staff allowed an up-to-date mental health review to be carried out by the new liaison team. Over time, increasing this overlap with primary care has been of considerable benefit to a number of residents with both medical and mental health needs. Similarly, close joint working with staff in primary care has led to the adoption of community DNAR orders in several cases after discussion with relevant family members and care home staff.

All the identified residents were then allocated a 'review frequency', which was compatible with the system of regular visits every 4 or 6 weeks. A convention was adopted for allocation of a visit in either the following month, in 3-4 months' time or after 6 months. To familiarise staff new to the process, these review categories were colour coded as red, orange and green respectively. Some residents were discharged from regular or routine follow-up and allocated to 'see on request'. Exceptionally, residents with active or acute mental health needs or changes in treatment required more frequent review and specific or individual arrangements were made accordingly.

All these decisions and changes were entered on the spreadsheets, which were updated on a monthly basis. As the team's experience and familiarity with this process developed, it became possible to identify in advance the level of need of each care home as a whole. Clearly, a small residential care home will have a lower level of overall need than a large general nursing home, particularly if there is an adjoining nursing facility providing care for residents with dementia, which is often the case in Bridgend.

\section{Results of the review process}

Comprehensive collation of a list of all the residents with mental health needs and allocation using the red, orange and green triage categories allowed the level of need to be quantified reasonably accurately and, more importantly, for a sufficient allocation of liaison staff for the next routine visit.

In terms of barrier analysis, the regime of monthly care home visits and the use of a reliable spreadsheet to anticipate the level of need are strong natural barriers, directly equivalent to the earlier examples given. The list of 'drugs of interest', which if seen simply as guidance would 
be classified as a (weak) administrative or paper barrier, has over time been used much more as a physical tactic (along with the spreadsheets) to establish a clear and consistent mental health approach and to reduce uncertainty and misunderstanding.

In a similar way, the use of a clearly written statement for each individual patient, describing the role and purpose of the antipsychotic medication (including diagnosis, target signs and symptoms and a firm commitment to a further mental health review), tends to be most effective when used routinely, as it then takes on the form of a natural or a physical intervention. Table 1 takes a wider perspective and shows in a summary grid the greater service value of these (stronger) physical and natural barriers.

The newly established liaison team identified unmet need within the care home sector for individual residents and in terms of care home staff training. This was associated with a small initial increase in appropriate admissions to hospital. However, ongoing analysis of statistics has confirmed a definite suppression of the total expenditure on continuing NHS care funding in this area. In turn, this is consistent with improved treatment for mental illness and earlier or more effective prevention provided to this patient group by the combined liaison service and training team approach.

As experience developed, it became possible to schedule visits to different care homes which are geographically close together, allowing individual mental health practitioners to provide advice and support more efficiently. This is especially efficient as it allows for frequent review or repeat assessments by different members of the team without disruption to the underlying programme of regular review. Following introduction of the team, the average rate of mental health admission from the care home sector was practically unchanged during the first 12 months, but reduced by a third to a sustained average of two admissions per month during the second year (for a population over 65 years of around 25000 ).

Eventually, it became possible to 'fix' a 4-weekly care home visit schedule, which was later relaxed to a more sustainable 6 -weekly cycle. The liaison team has affectionately christened this sequence the 'hamster wheel'. This will account for 48 weeks - the remaining 4 weeks each year have accordingly been identified as time off the wheel. Activities such as team building, audit and strategic review have usefully been held during these weeks, again avoiding unnecessary disruption to the cycle of regular care home visits.
TABLE 1 Summary of service interventions with respective barrier analysis category

\begin{tabular}{|lllll|}
\hline \multicolumn{2}{|c|}{$\begin{array}{c}\text { Orthodox or conventional sectorised } \\
\text { old age psychiatry service }\end{array}$} & & & \multicolumn{2}{c|}{$\begin{array}{c}\text { Combined education and liaison } \\
\text { old age psychiatry service }\end{array}$} \\
\cline { 1 - 2 } Strategy & Barrier & & Strategy & Barrier \\
\hline $\begin{array}{l}\text { Consultation model ('They } \\
\text { ask, we see') }\end{array}$ & Personnel & & $\begin{array}{l}\text { Single point of access for } \\
\text { referrals and reviews }\end{array}$ & Physical \\
\hline $\begin{array}{l}\text { No dedicated staff role } \\
\text { for training }\end{array}$ & Personnel & & $\begin{array}{l}\text { Allocation of dedicated } \\
\text { staff for training }\end{array}$ & Natural \\
\hline $\begin{array}{l}\text { Reliance on individual ad } \\
\text { hoc training }\end{array}$ & Personnel & & $\begin{array}{l}\text { Liaison model ('Looking for } \\
\text { problems') }\end{array}$ & Natural \\
\hline $\begin{array}{l}\text { Document-based advice } \\
\text { and guidance }\end{array}$ & Administrative & $\begin{array}{l}\text { Routine of regular } \\
\text { scheduled care home visits }\end{array}$ & Natural \\
\hline
\end{tabular}

Using the calendar to schedule routine visits to care homes provided an unexpected opportunity to find a solution to another perennial team management problem. The permanent liaison team is composed of mental health professionals drawn from a range of specialist backgrounds: medical (psychiatry), mental health nursing, psychology, social work and occupational therapy. (Administrative and clerical staff also make a vital contribution.) However, in a modern mental health service, the actual availability of individual members of staff is affected by a range of other demands. For example, mental health nursing staff are regularly called to contribute to continuing NHS healthcare assessments and decision support tool (DST) meetings to help to resolve difficult placement issues. Senior psychiatric staff are involved in a variety of sometimes complex medico-legal tasks, including formal roles under the Mental Health Act 1983 and the Mental Capacity Act 2005, often within certain specified timescales. More junior medical members of the team often have training commitments which cannot be altered and make a contribution to other rotas such as 'hospital at night' services.

Using a simple code for each post holder in the liaison team, an entry was made on each working day for each member of the team. If they were not available for mental health liaison team work on any given day, the code for that post was deleted. The week-to-week variation of staff availability was found to be immense even before sickness and absence is included.

With a little practice it was possible to establish a clear and coherent process using an electronic calendar to match the anticipated level of need in any given home with available staff from the liaison team. More importantly, in the event of a serious mismatch between need and the number of available staff, arrangements could be made with the care home to reschedule or to complete any remaining reviews on another occasion. 


EMI answers
$\begin{array}{llll}1 \mathrm{~g} & 2 \mathrm{c} & 3 \mathrm{f} & 4 \mathrm{a} \\ 5 \mathrm{k} & 60 & 7 \mathrm{i} & 8 \mathrm{p} \\ 9 \mathrm{~s} & 10 \mathrm{r} & 11 \mathrm{x} & 12 \mathrm{u}\end{array}$

Establishing this predictable routine then allowed the training team to coordinate their own complex educational demands with the clinical staff and achieve joint educational and clinical reviews. At a recent workshop in Bridgend attended by several care home managers, it was also identified that senior care staff and the managers themselves have been able to adjust their own duty rosters to coordinate with the liaison and training team visits - this collaborative approach received extremely positive evaluation and feedback from staff attending the workshop.

In future, the same transparent approach to scheduling will facilitate joint working with specialists outside the mental health field, ultimately to the benefit of the care home residents.

\section{Conclusions}

As part of a reorganisation of mental health services for older adults in Bridgend at the end of 2009, a new old age psychiatry liaison team has now become established, with a specific remit to deliver mental health services to residents in the care home sector as well as a traditional hospital liaison role.

The level of need for mental health support locally is substantial. There is ample evidence to predict that the current level of need will increase over time in Bridgend and more widely throughout the rest of England and Wales. Running parallel with this increasing need is a steadily rising demand for effective dementia care training.

In Bridgend, the improved level of service has been carefully coordinated and integrated with both an effective referral coordination system and an existing well-established dementia care training team. The highlights of our local experience are summarised in Box 3. In this article, barrier analysis has been useful to understand and to help explain the innovations and interventions introduced during the reorganisation.

The role of barrier analysis in reviews of adverse incidents is already well known - we believe that the same approach could be of great value to any mental health service, especially during a period of restructuring or planned reorganisation.

\section{Acknowledgements}

We would like to thank current and former members of the old age psychiatry liaison team and the two anonymous reviewers for their valuable contribution to the processes described in this article. We also thank Blood Bank manager Sylvia Lees at the Princess of Wales Hospital in Bridgend for her explanation of current blood transfusion procedures.
BOX 3 Effective strategies for care home liaison

DO allocate dedicated multiprofessional staff with an adequate range of experience to both training and service teams

DO ensure a comprehensive approach including all local care homes in service and training developments

$\mathrm{DO}$ ensure coordination between service and training teams

DO focus on repetition and reinforcement of teaching at an individual level within the care home sector

DO grasp opportunities to teach and train health and social care staff at all levels of experience in facilities that are fit for purpose

DON'T rely on an ad hoc teaching approach

DON'T assume that general classroom teaching will meet local or individual needs in the care home setting

Note: we support strategies that map most closely to the stronger physical and natural categories in barrier analysis rather than the weaker personnel and administrative categories.

\section{References}

All-Party Parliamentary Group on Dementia (2009) Prepared to Care: Challenging the Dementia Skills Gap. All-Party Parliamentary Group on Dementia.

Alzheimer's Research Trust (2010) Dementia 2010: The Economic Burden of Dementia and Associated Research Funding in the United Kingdom. University of Oxford.

Anonymous (2010) Does anyone care? BMJ 341: 348.

Ballard C, O'Brien J, James I, et al (2001) Dementia: Management of Behavioural and Psychological Symptoms. Open University Press.

Banks V, Searle G, Jenkins R (2011) Psychiatry in the UK: an overview. International Psychiatry 8: 35-8.

Bernstein CA, Hershfield B, Cohen DC (2010) Psychiatry in the USA: an overview. International Psychiatry 7: 90-2.

British Medical Association, Royal Pharmaceutical Society (2010) British National Formulary 59. BMJ Group \& Pharmaceutical Press.

Colgate R, Jones $S$ (2007) Controlling the confusion: management of referrals into mental health services for older adults. Advances in Psychiatric Treatment 13: 317-24.

Department of Health (2009) Living Well with Dementia: A National Dementia Strategy. TSO (The Stationery Office).

Godfrey M, Townsend J, Surr C, et al (2005) cited in Banks V, Searle G and Jenkins R (2011) Psychiatry in the UK: an overview. International Psychiatry 8: 35-8.

Jolley D, Dixey S, Read K (1998) Residential and nursing homes. In Seminars in Old Age Psychiatry (eds R Butler, B Pitt): 225-46. Gaskell.

Lawrence V, Bannerjee S (2010) Improving care in care homes: a qualitative evaluation of the Croydon care home support team. Aging and Mental Health 14: 416-24.

Lyons M, Woloshynowych M, Adams S, et al (2004) Error Reduction in Medicine. The Nuffield Trust National Patient Safety Agency, Imperial College London.

National Institute for Health and Clinical Excellence, Social Care Institute for Excellence (2006) Dementia. Supporting People with Dementia and 
their Carers in Health and Social Care (Amended 2011) (Clinical Guideline CG42). National Collaborating Centre for Mental Health.

Open University (2010) Interview. Critical care. Sesame 246: 15

Shaji KS, Dias A (2006) Dementia care in India: a progress report. International Psychiatry 4: 9-10.

Szczepura A, Nelson S, Wild D (2008) In-reach specialist nursing teams for residential care homes: uptake of services, impact on care provision and cost-effectiveness. BMC Health Services Research 8: 269.

Which? (2011) Care homes investigation. Which? (http://www.which. co.uk/campaigns/food-and-health/care-homes-investigation/which-carehomes-investigation/).

World Alliance for Patient Safety (2008) Surgical Safety Checklist. World Health Organization.

\section{EMls}

In each themed EMI, match the numbered statements $(1,2,3, \ldots)$ with a suitable response from the lettered options $(a, b, c, \ldots)$. Each option may be used once, more than once, or not at all and more than one option may be suitable for each statement.

\section{Theme: Dementia care training}

The dementia care training team has produced a series of modules to train health and social care staff.

1 Which of these modules is not one of the core sessions and was specially designed following a specific request?

2 Which module is usually the second session?

3 Which module would focus on depression in older people?

4 Which module considers the importance of maintaining mobility in the context of a resident in a care home?

Options

a Physical aspects of dementia care

b An overview of dementia

c Communication and dementia d Legal and ethical issues in dementia care

e Positive environments in dementia care

$f$ Mental health issues

g Pain in dementia

h Understanding behaviours in dementia

Theme: Barrier analysis

5 Which term is the natural barrier introduced by the World Health Organization to promote correct site surgery?

6 Which term describes a barrier that is often poorly effective?

7 Which term describes the current approach to safe blood transfusion by NHS Wales?

8 Which term describes the security layout of most commercial international airports?

Options

i Zero tolerance

j Sign in

k Time out

I Sign out

m Indelible marker

n Physical barrier

o Administrative barrier

p Geographical barrier

\section{Theme: Information technology}

9 Which item is used by the Bridgend old age psychiatry liaison team to schedule care home visits?

10 Which item is currently used by the team to record the list of residents with mental health needs in each care home?

11 Which item describes the process of colour coding and categorising the frequency of mental health reviews?

12 Which item describes the usual method of communication between the team and the referral coordinator?

\section{Options}

q Facsimile transmission

r Spreadsheet

s Calendar

t Database

u Email

v Search engine

w Social networking

$\mathrm{x}$ Triage 